\title{
Modification of Gate Dielectric in MOS Devices by Injection-Thermal and Plasma Treatments
}

\author{
V.V. Andreev ${ }^{a, *}$, G.G. Bondarenko ${ }^{b}$, V.M. Maslovsky ${ }^{c}$ And A.A. Stolyarov ${ }^{a}$ \\ ${ }^{a}$ Bauman Moscow State Technical University, Kaluga Branch, 2, Bazhenov Str., Kaluga, 248600, Russia \\ ${ }^{b}$ National Research University Higher School of Economics, 20, Myasnitskaya Ulitsa, Moscow 101000, Russia \\ ${ }^{c}$ The State Unitary Enterprise of a City of Moscow Research-and-Production Centre "SPURT" \\ Zelenograd, 4, West of the 1st proezd, 124460, Russia
}

\begin{abstract}
The influence of injection-thermal and plasma treatments on the characteristics of the MOS-structure is studied. It is shown that the thermal stable part of the negative charge which accumulates in the phosphorus-silicate glass (PSG) film in the structures with the two-layer gate dielectric $\mathrm{SiO}_{2}-\mathrm{PSG}$ under high-field Fowler-Nordheim electron injection can be used for the characteristics modification of MOS-structures with above described structure. The injection-thermal and plasma treatments of MOS-structures are offered to use for improving the reliability and finding the samples which have the charge defects. It is found that using the injection-thermal and plasma treatments allows to increase the injection and radiation stability of the dielectric films of MOS-structures due to structural changes in the $\mathrm{SiO}_{2}$ film and $\mathrm{Si}-\mathrm{SiO}_{2}$ interface.
\end{abstract}

DOI: $10.12693 /$ APhysPolA.125.1371

PACS: $73.40 . \mathrm{Qv}, 73.40 . \mathrm{Gk}$

\section{Introduction}

The influence of high-fields and ionizing radiation on the silicon MOS-structures has been intensively investigated in the middle of sixties of previous century [1]. The basic tendencies of investigation were connected with increase of charge and radiation stabilities of the MOS-devices and integrated circuits. Using the high-fields and radiation effects for control of the parameters of MOS devices has almost never been applied because of the significant degradation processes. However recently with design of new dielectric films and methods controlling their parameters, one creates possibility of the realization radiation and high-field treatments of MOS-structures for improvement and modification of their characteristics [1-3]. Creation of semiconductor devices with parameters which can be controlled after their manufacturing will let reduce nomenclature of manufactured semiconductor devices and integrated circuits in perspective using universal devices whose parameters can be changed after manufacturing both by the manufacturer and the consumer.

In this work the modification of MOS-structures using the high-field injection of electrons into the gate dielectric and the plasma treatments of the chips is studied. Also the time and temperature stability of the modified structures was estimated.

\section{Experimental techniques and samples}

Test MOS capacitors fabricated on Si $\langle 100\rangle n$-type wafers having resistivity of $4.5 \Omega \mathrm{cm}$ with thermal silicon dioxide and with $\mathrm{SiO}_{2}$ passivated by a phosphorus silicate glass (PSG) film were used. Silicon dioxide with

*corresponding author; e-mail: andreev@bmstu-kaluga.ru a thickness of $30-100 \mathrm{~nm}$ was thermally grown, using dry oxidation at $850 \div 1000^{\circ} \mathrm{C}$ with $3 \% \mathrm{HCl}$. PSG film was produced by doping of phosphorus from $\mathrm{POCl}_{3}-\mathrm{O}_{2}$ vapour at $900^{\circ} \mathrm{C}$. In order to obtain experimental samples with different PSG thickness, the phosphorus doping time ranged from 3 to 6 min. As gates with square dimensions of $10^{-4} \div 10^{-2} \mathrm{~cm}^{2}$, we used polysilicon ( $\mathrm{Si}^{*}$ ) film, $0.6 \mu \mathrm{m}$ thick, doped with phosphorus up to $20 \Omega \mathrm{m}^{-1}$ and aluminium film $1.2 \mu \mathrm{m}$ thick.

For injective modification of the electrophysical characteristics of MOS-structures there was used the high-field injection of electrons from silicon substrate at constant current stress with current density ranged from 0.1 to $10 \mu \mathrm{A} \mathrm{cm}^{-2}$ and at $20 \div 100{ }^{\circ} \mathrm{C}$ [4-6]. During the FowlerNordheim (FN) injection the voltage shift applied to the MOS structure characterizing the change in MOS structure charge state $[4,5]$ was measured. This allowed us to monitor changes of the MOS structure charge state during the process of modification. Also the change of charge state of MOS-structures is controlled using both high-frequency $C-V$ method and method of multilevel current stress [6]. To determine thermostable components accumulating negative charge in the gate dielectric after FN injection, MOS structures were annealed at a temperature of $200^{\circ} \mathrm{C}$ for a time from $200 \mathrm{~s}$ to $30 \mathrm{~min}$.

The radiofrequency ( $\mathrm{RF}$ ) treatment in standard manufacturing devices which has volume and diode reactors on the frequency $13.6 \mathrm{MHz}$ in oxygen plasma was used.

\section{Experimental results and discussions}

In previous articles $[5,6]$ it was shown that the negative charge which accumulates in PSG film of structure $\mathrm{Si}-\mathrm{SiO}_{2}-\mathrm{PSG}-\mathrm{Al}$ under the high-field tunneling electron injection can use for correction of the threshold voltage of these MOS-structures. Using measurements of both the thermally stimulated depolarization currents and isothermal relaxation $C-V$ characteristics at several fixed tem- 
peratures it is determined that under the high-field electron injection in $\mathrm{Si}-\mathrm{SiO}_{2}-\mathrm{PSG}-\mathrm{Al}$ structures accumulated negative charge consists of the two components. The first component of charge has a low thermal stability and it flows during the annealing at $200{ }^{\circ} \mathrm{C}$. The second component of charge has a high thermal stability and starts to flow at $230^{\circ} \mathrm{C}$ or more. Thus for receiving devices with high thermal stability after their modification under the high-field electron injection, an annealing at $200{ }^{\circ} \mathrm{C}$ is needed.

Figure 1 showed dependences of drift threshold voltage MOSFET after FN injection from silicon substrate (curve 1) and following sample annealing (curve 2) versus time of injection. For realization of the mode of high-field electron injection from the silicon substrate to the gate of MOSFET, the $1 \mu \mathrm{A} / \mathrm{cm}^{2}$ constant current pulse was applied.

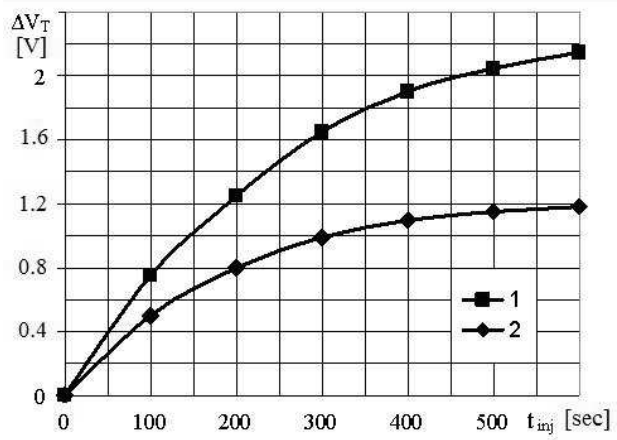

Fig. 1. The time dependence of the shift threshold voltage MOSFET under the high-field electron injection from the silicon substrate (curve 1) and followed by annealing (curve 2).

Figure 1 (curve 2) characterizes the thermal stable component of the negative charge. For receiving this component after the injection, modification of MOSFET was annealed at $200{ }^{\circ} \mathrm{C}$ during $20 \mathrm{~min}$. As can be seen in Fig. 1, thermal stable component of the negative charge is about $60 \%$ of total density of charge which accumulated in the gate dielectric under the FN injection.

Other way for applying high-field injection for modification dielectric films of MOS-structures is using injection thermal treatment which lets to increase reliability of MOS-devices and finding the samples which have charging defects. The injection thermal treatment of MOS-structures represents the high-field injection in dielectric with the determined charge density of electrons and following high temperature $\left(200^{\circ} \mathrm{C}\right)$ annealing of samples. In Refs. [1, 5] there has been shown the similarity of most of degradation processes of MOS-structures which occurred as under the ionizing radiation so under the high-field. Using the high-field injection instead radiation thermal treatment really simplifies testing process and also lets to receive large volume of additional information using control of modification of charging state of gate dielectric under the FN high-field.

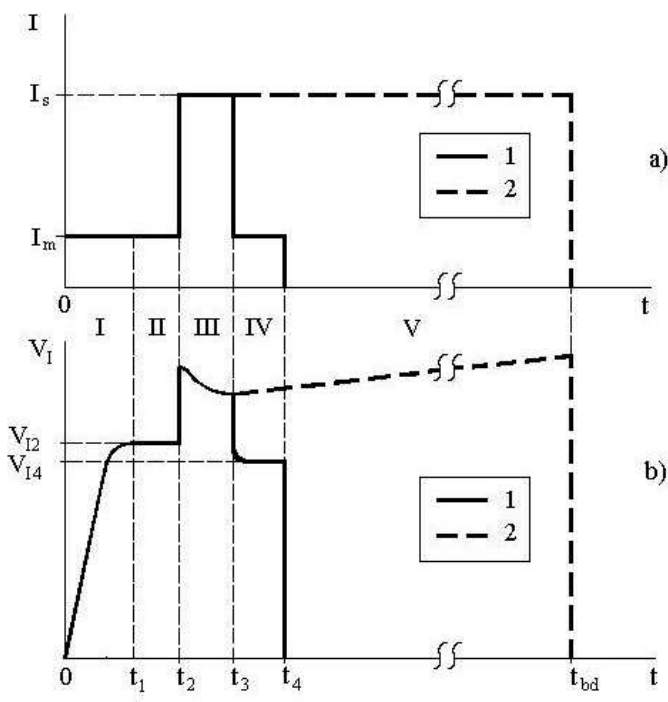

Fig. 2. The time dependences of the flowing current and voltage applied to the MOS structure in conditions of injection-thermal treatment (1) and testing the injected charge-to-breakdown (2).

Figure 2 shows the time dependences of the current and voltage of MOS structures in the mode of injection-thermal treatment (1) and measuring the injected charge to breakdown (2). Section I in Fig. 2 corresponds to the charge capacity of the MOS structure constant current $I_{\mathrm{m}}$. In this section all the current flowing through the dielectric is capacitive. In sections II and IV the charge injection into the dielectric carries out the measuring constant current $I_{\mathrm{m}}$. Amplitude of the current $I_{\mathrm{m}}$ should be much smaller than the amplitude of stress current $I_{\mathrm{s}}$, to achieve the correct measurement of the change of the charge state of the MOS structure after injection thermal treatment (ITT) [4].

Controlling of the amount of charge injected into the dielectric to breakdown, as a rule, was carried out at the amplitude of the current pulse $I_{\mathrm{S}}$ (Fig. 2, section V).

Figure 3 shows the histograms of MOS structures distribution by the charge-to-breakdown for the samples, illustrating the influence of injection-thermal treatment. Histogram 1 in Fig. 3 is for the group of structures without the ITT and histogram 2 corresponds to the group of MOS structures after ITT.

It could be seen in the histogram (Fig. 3) that the ITT allows to detect and eliminate the structures with coarse defects of isolation. After the treatment practically there are no structures with low (lower than $1 \mathrm{mC} / \mathrm{cm}^{2}$ ) charge-to-breakdown. Herewith, the injection resource of the samples does not decrease and this is a positive fact in the course of ITT. As a result of the application of ITT, a significant increase in injection and radiation stability of the structures occurs (Fig. 3). It can be explained by the formation of more perfect structure of the gate dielectric, because the high-field injection of electrons breaks the strained bonds in $\mathrm{SiO}_{2}$ film and on $\mathrm{Si}-\mathrm{SiO}_{2}$ interface, 


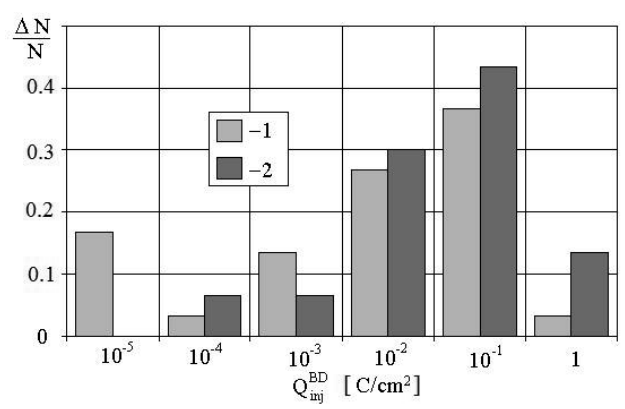

Fig. 3. Histograms of MOS structures distribution by the injected charge-to-breakdown for the samples without treatment (1) and after injection-thermal treatment (2).

and the subsequent annealing restores the valence bonds even without their deformation [1-3].

It is shown that the ITT at elevated temperatures can lead to the degradation of reliability characteristics of MOS devices. This phenomenon seems to be associated with problem of the activation of electron and hole traps at elevated temperatures. It is shown that in order to reduce the probability of gate dielectric breakdown and efficiency ITT the better high-field charge injection is at room temperature. The use of high-field injection instead of radiation treatments [7] simplifies the testing process and provides a great deal of additional information at monitoring of the change in the charge state of the gate dielectric during the high-field injection. Therefore, the performance of ITT can improve the injection and radiation of the resistance of gate dielectric in MOS devices.

It was found that under some modes of the plasma treatment, the density and the rate of generation of new electronic traps in the silicon dioxide generated during the high-field electrons injection can be greatly reduced. This can greatly improve the charge stability of the structure and increase such an important parameter as the value of injected charge-to-breakdown [5].

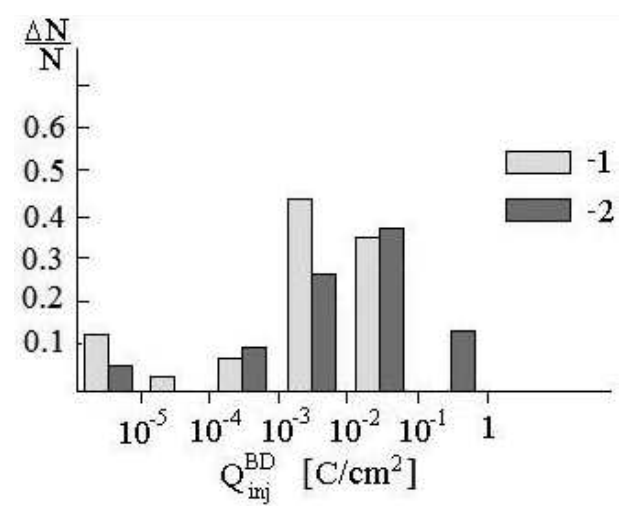

Fig. 4. Histograms of MOS structures distribution by the injected charge-to-breakdown for the samples without treatment (1) and after plasma treatment (2).
Figure 4 shows the histograms of MOS structures distribution by the value of the injected charge-to-breakdown for the samples without treatment (1) and after plasma treatment (2). As can be seen from Fig. 4, after the plasma treatment the maximum number of the injected charge-to-breakdowns can be increased considerably.

The required density of electron traps in the volume of $\mathrm{SiO}_{2}$ film under some technological modes of RF plasma treatment of MOS structures can be created. As a result, at the initial stage of FN high-field electron injection the more dominant process of the change of charge state of the gate dielectric is the electron capture in the film $\mathrm{SiO}_{2}$ comparing with the generation of a positive charge in the structures without treatment. This reduces the probability of breakdown of the samples. After the capture of negative charge on the created plasma electronic traps, the value of the potential barrier at the injection interface which leads to the reduction of local currents flowing in the defects and, consequently, to the reduction of the possibility of the samples breakdown was increased.

\section{Conclusions}

It is determined that the negative charge, accumulating in PSG film in structures with two-layer gate dielectric $\mathrm{SiO}_{2}-\mathrm{PSG}$ under the high-field electron injection, can be used for the modification of electrophysical characteristics of MOS-devices. It was shown that the ITT can improve the MOS structures reliability, leading to the increase of the charge injected to breakdown, and can identify structures containing defects. The plasma treatment also can improve the characteristics of MOS-devices, increasing the injection and radiation stability of the gate dielectric.

\section{Acknowledgments}

This work was supported by the Ministry of Education and Science of the Russian Federation, the RFBR and the administration of Kaluga region (grant No. 12-02-97533) and the study was implemented in the framework of the Basic Research Program of the National Research University Higher School of Economics (HSE).

\section{References}

[1] S. Lombardo, J.H. Stathis, P. Linder, K.L. Pey, F. Palumbo, C.H. Tung, J. Appl. Phys. 98, 121301 (2005).

[2] V.V. Afanas'ev, A. Stesmans, J. Appl. Phys. 102, 081301 (2007).

[3] V.A. Gritsenko, Physics-Uspekhi 52, 869 (2009).

[4] V.V. Andreev, G.G. Bondarenko, V.M. Maslovsky, A.A. Stolyarov, IOP Conf. Series: Mater. Sci. Eng. 41, 012017 (2012).

[5] G.G. Bondarenko, V.V. Andreev, V.M. Maslovsky, A.A. Stolyarov, V.E. Drach, Thin Solid Films 427, 377 (2003).

[6] G.G. Bondarenko, V.V. Andreev, V.E. Drach, S.A. Loskutov, M.A. Stolyarov, Thin Solid Films 515, 670 (2006).

[7] United States Military Standard MIL-STD-883H Method 1019.8. 